\title{
Application of Recent Developments in Deep Learning to ANN-based Automatic Berthing Systems
}

\author{
Daesoo Lee, Seung-Jae Lee*, Yu-Jeong Seo \\ Division of Naval Architecture and Ocean Systems Engineering, Korea Maritime and Ocean University, Busan, Korea \\ Received 31 May 2019; received in revised form 21 June 2019; accepted 16 November 2019 \\ DOI: https://doi.org/10.46604/ijeti.2020.4354
}

\begin{abstract}
Previous studies on Artificial Neural Network (ANN)-based automatic berthing showed considerable increases in performance by training ANNs with a set of berthing datasets. However, the berthing performance deteriorated when an extrapolated initial position was given. To overcome the extrapolation problem and improve the training performance, recent developments in Deep Learning (DL) are adopted in this paper. Recent activation functions, weight initialization methods, input data-scaling methods, a higher number of hidden layers, and Batch Normalization $(\mathrm{BN})$ are considered, and their effectiveness has been analyzed based on loss functions, berthing performance histories, and berthing trajectories. Finally, it is shown that the use of recent activation and weight initialization method results in faster training convergence and a higher number of hidden layers. This leads to a better berthing performance over the training dataset. It is found that application of the $\mathrm{BN}$ can overcome the extrapolated initial position problem.
\end{abstract}

Keywords: automatic ship berthing, artificial neural network, deep neural network, batch normalization

\section{Introduction}

When a ship approaches a port or harbor, which is called a "berth operation," slow speeds cause nonlinear characteristics in a ship's motion, and the ship undergoes sudden changes in its rudder angle and engine revolutions per second (RPS). For this reason, unlike ship navigation, where ships sail almost at a constant speed, the ship berthing problem is a challenging one. In order to overcome these difficulties, many intelligent control algorithms have been proposed, and one of them is the Artificial Neural Network (ANN) or simply the Neural Network (NN). With the rapid development of Deep Learning (DL), systems based on neural networks have drastically increased and demonstrate extraordinary performance. Therefore, many studies on the application of neural networks to ship berthing systems have been conducted.

Im and Hasegawa [1] proposed the "Parallel Neural Network," which makes the neural network focus on each task by changing the ordinary neural network architecture. Im and Hasegawa [2] attempted to use another neural network to identify ship motions so that the neural network system could cope with wind disturbances. Im [3] proposed an algorithmic method using a neural network to allow ships to berth safely, even when they approached from unusual directions. Bae et al. [4] compared two neural networks with different input configurations and showed that both input configurations led to a similar performance. Ahmed and Hasegawa [5] used a virtual window concept and nonlinear programming method to generate teaching data consistently. Im and Nguyen [6] tried solving the problem in which a neural network trained with a ship berthing dataset at one port can only be used at that particular port but not at others. The researchers adopted a head-up coordinate system instead of a north-up coordinate system, and the head-up coordinate system allowed the neural network trained with a ship berthing dataset at one port to be used at another port as well.

* Corresponding author. E-mail address: slee@kmou.ac.k 
However, although many studies on ANN-based automatic berthing have been conducted to date, they have missed to address several important issues. First, they neither considerd to use recent activation functions nor mentioned about weight initialization methods. Second, they used an inefficient optimizer, although, there are other optimizers with higher performance that have been proposed and verified. Third, there are no consistent method for input data scaling, and some previous studies scaled their input data in inefficient ways. Fourth, no regularization methods can prevent the overfitting problem have been considered. Fifth, owing to the overfitting problem caused by the lack of a regularization method, poor berthing performance occurred when neural networks were given extrapolated initial positions. Therefore, most of the previous studies showed berthing trajectories with initial positions from training datasets and interpolated or slightly extrapolated initial positions only.

In this paper, recent activation functions, weight initialization methods, optimizers, input scaling methods, neural networks with a higher number of hidden layers, and Batch Normalization (BN) proposed by Loffe and Szegedy [23] are introduced and applied to neural network models. Their effectiveness is verified based on the loss functions, berthing performance histories and berthing trajectories.

\section{Mathematical Model of Ship Maneuvering}

To build a mathematical model of ship maneuvering, a definition of a coordinate system and principal particulars of a ship are first presented in Fig. 1 and Table 1, respectively. And then, a mathematical model of ship maneuvering and modeling of a propeller and rudder are presented in the following two subsections.

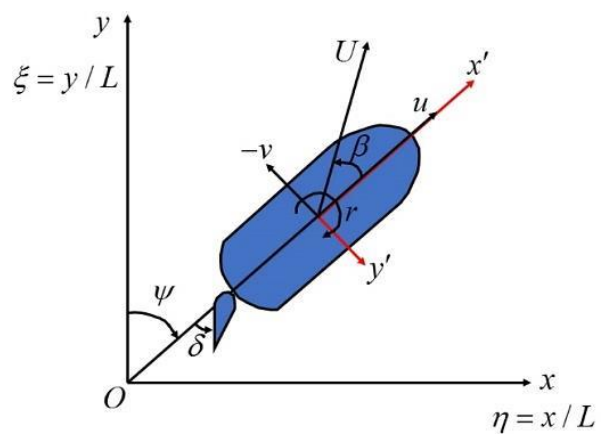

Fig. 1 Coordinate system

where $x$ and $y$ are the actual positions of the ship in meters, and $\eta$ and $\xi$ are the normalized positions of $x, y$ by the length of the ship.

Table 1 Principal particulars of a target ship

\begin{tabular}{|c|c|c|}
\hline \multicolumn{3}{|c|}{ HULL } \\
\hline Length overall & $L_{O A}[\mathrm{~m}]$ & 188.0 \\
\hline Length between perpendiculars & $L[\mathrm{~m}]$ & 175.0 \\
\hline Breath & $B[\mathrm{~m}]$ & 25.4 \\
\hline Draft & $d[\mathrm{~m}]$ & 8.50 \\
\hline Block coefficient & $C_{B}$ & 0.559 \\
\hline \multicolumn{3}{|c|}{ RUDDER } \\
\hline Height & $H_{R}$ & 7.70 \\
\hline Area ratio & $A_{R} /(L d)$ & $1 / 45.8$ \\
\hline Aspect ratio & $\lambda$ & 1.827 \\
\hline PROPELLER \\
\hline Diameter & $D[\mathrm{~m}]$ & 6.5 \\
\hline Pitch ratio & $P / D$ & 1.055 \\
\hline Expanded area ratio & & 0.730 \\
\hline
\end{tabular}




\subsection{Mathematical model for ship-maneuvering problem}

In the ship-maneuvering problem, a planar motion includes only surge, sway and yaw motions, which are considered out of six-degree-of-freedom motions. The basic equations of motion for ship maneuvering can be expressed as follows:

$$
\begin{aligned}
& m(\dot{u}-v r)=F_{\text {surge }} \\
& m(\dot{v}+u r)=F_{\text {sway }} \\
& I_{z} \dot{r}=M_{\text {yaw }}
\end{aligned}
$$

where $m$ is the mass, and $u, v, r$ are the velocities in the surge, sway, yaw directions, respectively. $I_{z}$ is the moment of inertia with respect to the $z$-axis, and $F_{\text {surge }}, F_{\text {sway }}, F_{\text {yaw }}$ are the surge and sway forces and yaw moment, respectively. The dot represents the time derivative. The right terms in Eqs. (1)-(3) can be dealt with using the established procedure from Newman [24], and Eqs. (1)-(3) can be expressed as Eqs. (4)-(6).

$$
\begin{aligned}
& \left(m+m_{x}\right) \dot{u}-\left(m+m_{y}\right) v r=X \\
& \left(m+m_{y}\right) \dot{v}+\left(m+m_{x}\right) u r=Y \\
& \left(I_{z}+J_{z}\right) \dot{r}=N-x_{G} Y
\end{aligned}
$$

where $m_{x}, m_{y}, J_{z}$ are the added mass in the surge and sway directions and the added moment of inertia, respectively. $X, Y$ are the hydrodynamic forces, and $N$ is the hydrodynamic moment. $x_{G}$ is the distance from the midship to the center of gravity. The hydrodynamic forces and moment are generally expressed as Eqs. (7)-(9), which were proposed by the Maneuvering Modeling Group (MMG).

$$
\begin{aligned}
& X=X_{H}+X_{P}+X_{R}+X_{W}+X_{T} \\
& Y=Y_{H}+Y_{P}+Y_{R}+Y_{W}+Y_{T} \\
& N=N_{H}+N_{P}+N_{R}+N_{W}+N_{T}
\end{aligned}
$$

where subscripts $H, P, R, W, T$ refer to the hull, propeller, rudder, wind and tug, respectively. However, due to this paper focuses on the application of recent developments in DL; the external forces such as the wind and tug are not considered.

A ship's maneuvering displacements and velocities can be calculated numerically by integrating Eq. (10)-(12) using the Runge-Kutta $4^{\text {th }}$-order method.

$$
\begin{aligned}
& \dot{u}=\frac{X+\left(m+m_{y}\right) v r}{\left(m+m_{x}\right)} \\
& \dot{v}=\frac{Y-\left(m+m_{x}\right) u r}{\left(m+m_{y}\right)} \\
& \dot{r}=\frac{N-x_{G} Y}{I_{z}+J_{z}}
\end{aligned}
$$

\subsection{Modeling of propeller and rudder}

Owing to the kinematics of the mechanical system of the rudder and engine, time delays between commands of operation and the resultant mechanical response may occur. The equations from Shon [13] to consider this physical time-delay effect are used here. First, the equation to consider the time-delay effect for the propeller RPS is Eq. (13). 


$$
\dot{n}=\left(n^{*}-n\right) / T_{n}
$$

where $n$ is the RPS, $\dot{n}$ is a derivative of RPS with respect to time, and $n^{*}$ is the target RPS. $T_{n}$ is a time-delaying constant for the RPS, and this variable is set to $15 \mathrm{~s}$ in this paper. Next, equations to consider the time-delay effect for a rudder are Eqs. (14)-(15).

$$
\begin{aligned}
& \dot{\delta}=\left(\delta^{*}-\delta\right) / T_{E} ;\left|\delta^{*}-\delta\right| \leq T_{E}\left|\dot{\delta}_{\max }\right| \\
& \dot{\delta}=\operatorname{sign}\left(\delta^{*}-\delta\right)\left|\dot{\delta}_{\max }\right| ;\left|\delta^{*}-\delta\right|>T_{E}\left|\dot{\delta}_{\max }\right|
\end{aligned}
$$

where $\delta^{*}$ is the target rudder angle, $T_{E}$ is the time-delaying constant for the rudder, and $\dot{\delta}_{\max }$ is the maximum angular velocity of the rudder. In this paper, $T_{E}$ is set to $2.5 \mathrm{~s}$, and $\dot{\delta}_{\max }$ is set to $3.0 \%$ s.

\section{Artificial Neural Network and Important Factors in Training the Network}

\subsection{Artificial neural network}

The basic architecture of the neural network used in this paper is illustrated in Fig. 2, $\eta$ is $x / L$, and $\xi$ is $y / L$, in which $x, y$ are the relative positions of the ship from the target berthing point, and $L$ is the LBP of the ship. $u, v, r$ are the velocities of the ship in the surge, sway, and yaw directions, respectively, and $\psi$ is the heading angle of the ship. In the output layer, $\delta_{t \text { arget }}, n_{t \text { arget }}$ are the target rudder angle and target RPS, respectively. This input layer configuration has been used in several papers such as those of Im and Hasegawa [1] and Ahmed and Hasegawa [5]. The number of hidden layers and the size of the neural network will be addressed in Section 3.5.

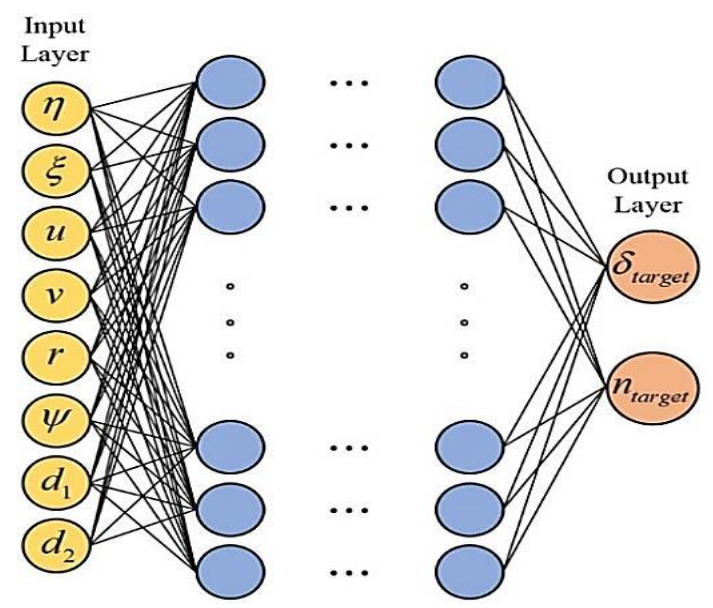

(a)

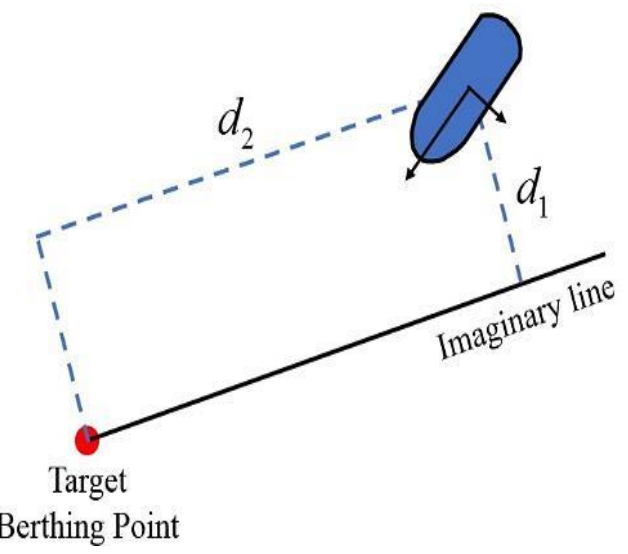

(b)

Fig. 2 Basic architecture of neural network, and concept of imaginary line

The imaginary line shown in Fig. 2 is used as a guideline during berthing. It is known that as the imaginary line provides the additional input variables of $d_{1}$ and $d_{2}$, which can improve the berthing performance by the neural network. The angle between the $x$-axis and the imaginary line is set to $20^{\circ}$ in this paper.

For the teaching data preparation, the patterns of the berthing trajectories, rudder angle, and RPS control from Nguyen and $\operatorname{Im}$ [14] and Bae et al. [4] are referred. The teaching dataset used in this paper is shown in Fig. 3, where the red crosses are the initial positions in the teaching data, the target berthing facility is drawn in blue, and the orange straight line is the imaginary line. 


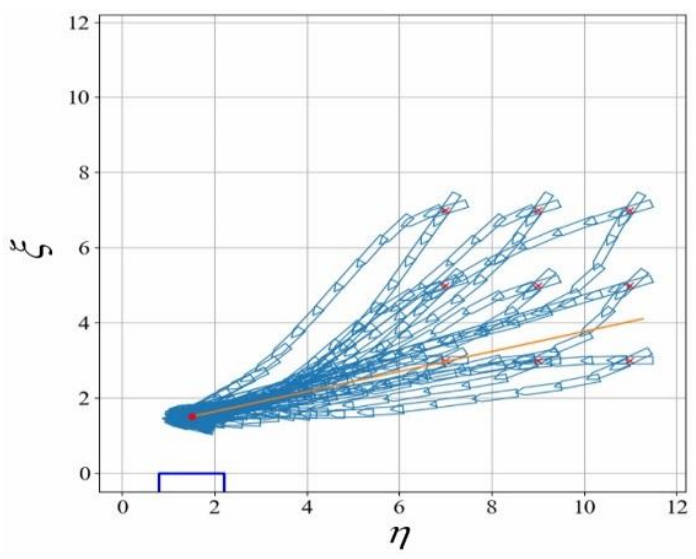

Fig. 3 Teaching dataset

The end of the berthing operation is defined as a status meeting and the following three conditions according to Ahmed and Hasegawa [15]. First, the aim is to berth at a distance of around the length of 1.5 times farer than where the ship is from the berthing facility. Second, the relative heading angle to the berthing facility should be smaller than $30^{\circ}$. Third, the velocity of the ship should be slower than $0.2 \mathrm{~m} / \mathrm{s}$.

The overall flowchart for the automatic ship berthing with a neural network is shown in Fig. 4, the left side of the dotted line is the preparation step for the neural network model, and the right side is a processing loop with the trained neural network. In this paper, building and training the neural networks are carried out with the open-source software library Tensorflow developed by the Google Brain Team.

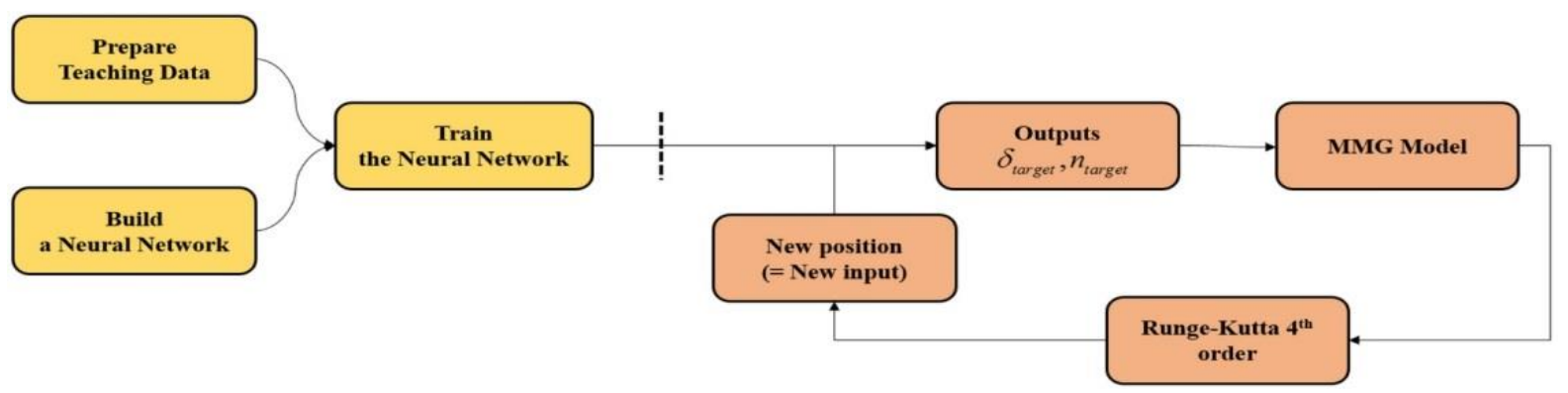

Fig. 4 Flowchart for ANN-based ship berthing system

\subsection{Activation function and weight initialization}

Choosing both an appropriate activation function and weight initialization method is quite important in building a neural network. The training performance and training speed can be greatly affected by these two parameters.

For the activation function, many previous studies such as those of Im [3], Bae et al. [4], Ahmed and Hasegawa [5], and Lee and Lee et al. [26] used the sigmoid function. However, it is known that the sigmoid function intrinsically has some disadvantages in training neural networks. According to Nwankpa et al. [25], the drawbacks of the sigmoid function are sharp damp gradients during backpropagation, gradient saturation, slow convergence, and nonzero-centered output. Thus, many studies have been carried out to develop better activation functions to overcome the problems of the sigmoid function. The activation functions that are popularly used these days are the tangent hyperbolic and Rectified Linear Unit (ReLu) functions proposed by Nair and Hinton [7]. The tangent hyperbolic function solves the problem of the limitation in the gradient update direction that exists in the sigmoid function, and results in a faster training speed. The ReLu function avoids the gradient vanishing problem and lowers the complexity of the calculations as its derivative function is relatively simple. The equations for the sigmoid, tangent hyperbolic, and ReLu functions are shown in Eqs. (16)-(18):

$$
\operatorname{sigmoid}(x)=1 /\left(1+e^{-x}\right)
$$




$$
\begin{gathered}
\tanh (x)=\frac{e^{x}-e^{-x}}{e^{x}+e^{-x}} \\
\left\{\begin{array}{l}
\operatorname{relu}(x)=x ; \quad x \geq 0 \\
\operatorname{relu}(x)=0 ; \text { otherwise }
\end{array}\right.
\end{gathered}
$$

For the weight initialization method, the normal distribution with a mean of zero and a standard deviation of 0.01 was mostly used. Later, however, many studies showed that the weight initialization method can greatly affect the training performance, and many other weight initialization methods have been proposed. Among the proposed methods, the two most popular at present are the Xavier initialization (also called the Glorot initialization) by Glorot and Bengio [16] and the He initialization by $\mathrm{He}$ et al. [17]. According to Glorot and Bengio [16], the Xavier initialization results in good training performance with the sigmoid and tan $h$ functions. According to He et al. [17], the He initialization is very compatible with the ReLu function. The equations for the Xavier and He initialization methods are based on a normal distribution where a mean value is set to zero and variance is set to Eq. (19) for the Xavier initialization and Eq. (20) for the He initialization.

$$
\begin{aligned}
& \sigma_{\text {Xavier }}^{2}=\sqrt{2 /\left(n_{\text {in }}+n_{\text {out }}\right)} \\
& \sigma_{\mathrm{He}}^{2}=\sqrt{2 / n_{\text {in }}}
\end{aligned}
$$

where $n_{\text {in }}$ and $n_{\text {out }}$ represent the numbers of the previous and next layer's nodes.

\subsection{Optimizer}

In previous studies, such as those of Im and Hasegawa[1], Im and Hasegawa [2], Im [3], Ahmed and Hasegawa [5], and Im and Nguyen [6] adopted the Levenberg-Marquardt approach proposed by Levenberg [9]. However, according to Bazzi et al. [10], who analyzed the training performance of several popular optimizers including Levenberg-Marquardt, the Root Mean Square Propagation (RMSProp) method proposed by Tieleman and Hinton [11] is faster and computationally more efficient, and requires fewer hyperparameters than Levenberg-Marquardt. Furthermore, Kingma and Ba [12] showed that the Adam optimizer proposed by Kingma and Ba [12], which is a combination of RMSprop and Momentum proposed by Rumelhart et al. [19], outperforms RMSprop. Therefore, Adam is adopted as an optimizer in this paper. The learning rate of the Adam is set to 1e-3, which results in a robust training performance in general. For the loss function to be minimized by Adam, the Mean Square Error (MSE) is used.

\subsection{Input data scaling}

Table 2 List of input data-scaling methods

\begin{tabular}{|l|c|c|}
\hline Scaling \#1 & 0 to 1 Min-Max Scaling & Scales inputs to range of 0 to 1 \\
\hline Scaling \#2 & -1 to 1 Min-Max Scaling & Scales inputs to range of -1 to 1 \\
\hline Scaling \#3 & Standard Scaling & Standardizes inputs by removing mean and scaling to unit variance \\
\hline
\end{tabular}

Because the input data of a ship berthing dataset for neural network contains variables of different units, the weights are likely to be updated in an unstable way during training when input data scaling is not applied because of the different scales of each input variable. However, among the previous studies on ANN-based automatic berthing, some did not employ a scaling method for their input data, and some applied a scaling method on some input variables only. A few of the studies scaled the input data to between 0 and 1. The commonly used input data-scaling methods are presented in Table 2 and Eqs. (21)-(23).

$$
\begin{aligned}
& x^{\prime}=(x-\min (X)) /(\max (X)-\min (X)) \\
& x^{\prime}=(x-2 \times \min (X)) /(\max (X)-\min (X))
\end{aligned}
$$




$$
x^{\prime}=(x-\operatorname{mean}(X)) / \operatorname{std}(X)
$$

where $x$ is the sampled data, $x^{\prime}$ is the scaled sampled data, and $X$ is a set of data.

\subsection{Number of hidden layers}

The number of hidden layers has a close relationship with the neural network model's system capacity. It is commonly known that if a neural network model has a large number of hidden layers; it has a large capacity to understand the complexity of a given training dataset and to have a complex system. However, simply using many hidden layers does not guarantee the neural network's good performance. Numbers of hidden layers of 5, 10, 20, 30, and 40 are considered and investigated in Section 4. The hidden layer size is also an important factor in the neural network architecture, but in order to build a neural network model for a complex system, the number of hidden layers is more influential. Generally, the hidden layer size is set to $2^{n} . n$ is 5 for simple systems, 6 for a slightly complex systems, and 8-9 for complex systems. In this paper, the hidden layer size is set to $2^{6}$.

\subsection{Overfitting prevention}

Algorithm 1 Batch Normalization

Input: Values of $x$ over a mini-batch: $\mathrm{B}=\left\{x_{1 \ldots \mathrm{m}}\right\} ;$ Parameters to be learned: $\gamma, \beta$
Output: $\left\{y_{i}=\mathrm{BN}_{\gamma, \beta}\left(x_{i}\right)\right\}$
$\mu_{\beta} \leftarrow \frac{1}{m} \sum_{i=1}^{m} x_{i} \quad$ // mini-batch mean
$\sigma_{\beta}^{2} \leftarrow \frac{1}{m} \sum_{i=1}^{m}\left(x_{i}-\mu_{\beta}\right)^{2} \quad / /$ mini-batch variance
$\hat{x} \leftarrow \frac{x_{i}-\mu_{\beta}}{\sqrt{\sigma^{2}+\varepsilon}} \quad$ // normalize
$y_{i} \leftarrow \gamma \hat{\mathrm{x}}_{i}+\beta \equiv \mathrm{BN}_{\gamma, \beta}\left(x_{i}\right) \quad / /$ scale and shift

Overfitting may occur when a neural network is trained with a given training dataset over many epochs because the weights become too fit to the given dataset only. Then, the performance of the neural network fails with new input data. In an automatic berthing using a neural network, if overfitting occurs, the neural network will show poor berthing performance when a new initial position is given. The poor performance with the overfitting issue occurs more clearly when new input data is extrapolated rather than interpolated by the neural network. In previous studies on ANN-based automatic berthing, no regularization methods to prevent overfitting were considered. Some papers such as Bae et al. [4] showed that poor berthing performance occurs owing to an extrapolated initial position. In order to deal with the poor performance caused by the extrapolation, the $\mathrm{BN}$ is adopted. The advantages of the $\mathrm{BN}$ are as follows. It allows for a higher learning rate, reduces the dependence of initial weights, and provides a regularization effect to prevent overfitting. The pseudocode of BN is shown in Algorithm 1. Where $\varepsilon$ is a small value of about $10^{-6}$ to prevent division by zero.

\section{Application of Recent Developments in Deep Learning to Automatic Berthing}

In this section, the effects of activation functions, weight initialization methods, input data-scaling methods, numbers of hidden layers, and the BN that were introduced in the previous section are investigated in terms of the neural network's training performance and berthing performance. 
As to the activation functions and weight initialization methods, nine different neural network models with nine combinations from three activation functions of the sigmoid, tangent hyperbolic $(\tan h)$, and ReLu; and three weight initialization methods of the normal distribution, Xavier, and He are built. In order to show the training progress over epochs, a loss function history graph is presented in Fig. 5. Details of the training models are listed in Table 3 for better reproducibility.

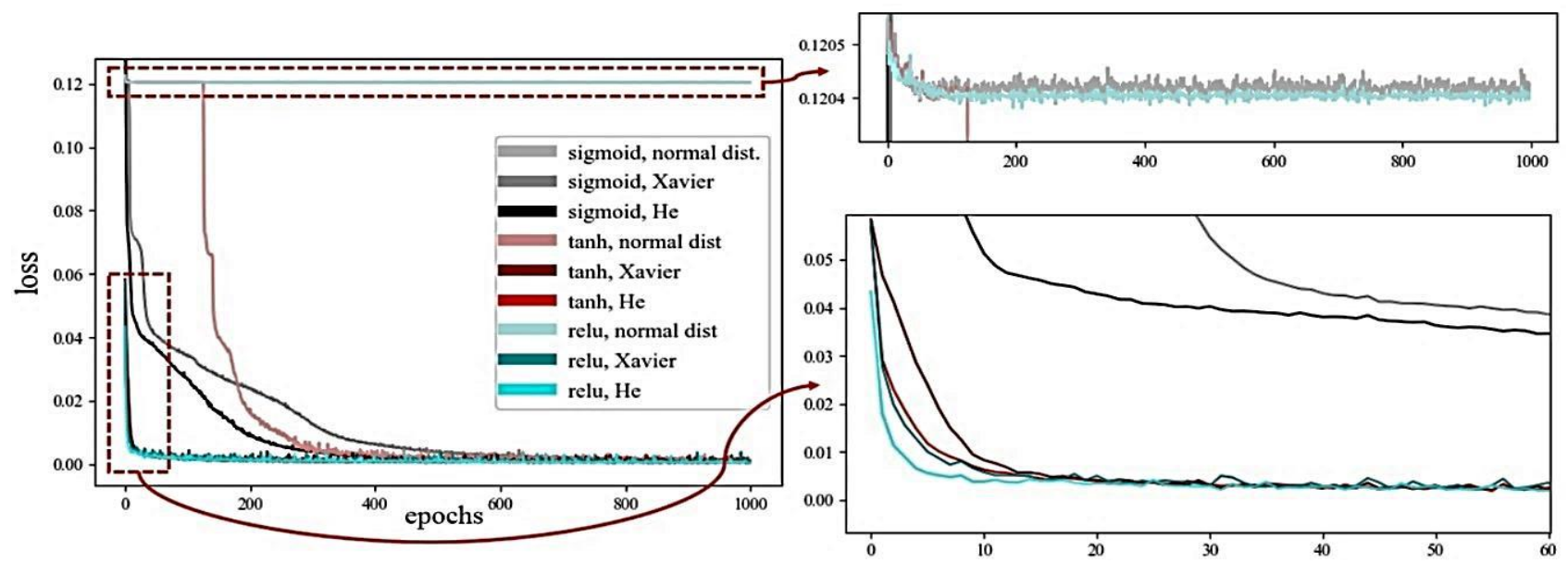

Fig. 5 Loss function histories with respect to activation and weight initialization methods

Table 3 Details of training models with respect to activation and weight initialization methods

\begin{tabular}{|c|c|c|c|c|c|c|c|}
\hline $\begin{array}{c}\text { Hidden } \\
\text { layer } \\
\text { size }\end{array}$ & $\begin{array}{c}\text { Number of } \\
\text { hidden layers }\end{array}$ & $\begin{array}{c}\text { Optimizer, } \\
\text { learning rate }\end{array}$ & $\begin{array}{c}\text { Number of } \\
\text { epochs }\end{array}$ & $\begin{array}{c}\text { Batch } \\
\text { size }\end{array}$ & $\begin{array}{c}\text { Activation } \\
\text { function }\end{array}$ & Weight initialization & $\begin{array}{c}\text { Input } \\
\text { data-scaling } \\
\text { method }\end{array}$ \\
\hline $2^{6}$ & 10 & $\begin{array}{c}\text { Adam, } \\
1 \mathrm{e}-3\end{array}$ & 1000 & $2^{7}$ & $\begin{array}{c}\text { sigmoid, } \\
\text { tanh, ReLu }\end{array}$ & $\begin{array}{c}\text { normal distribution, } \\
\text { Xavier, He }\end{array}$ & $\begin{array}{c}-1 \text { to } 1 \mathrm{Min}-\mathrm{Max} \\
\text { Scaling }\end{array}$ \\
\hline
\end{tabular}

The number of hidden layers is set to 10 to make use of Deep Neural Networks (DNNs). The effect of the number of hidden layers is investigated later in this section. The batch size is set to $2^{7}$ because this is usually set to $2^{n}$, where $n$ is generally 5 to the minimum and 10 to the maximum.

In Fig. 5, the models with weight initialization by normal distribution led to a slow training speed and poor convergence. The models using the sigmoid function show a slow training speed as well. It is shown that the traditional activation function and weight initialization method are inefficient in terms of training the DNN for automatic ship berthing. The best combination of the activation function and weight initialization method is ReLu-He. He et al. [17] claimed that the ReLu function is very compatible with He weight initialization.

For the input data-scaling methods, the effect of each input data-scaling method is analyzed with a loss function history. The loss function history is shown in Fig. 6, and details of the training models with respect to the input data-scaling method are listed in Table 4.

Table 4 Details of training models with respect to activation and weight initialization methods

\begin{tabular}{|c|c|c|c|c|c|c|c|}
\hline $\begin{array}{c}\text { Hidden } \\
\text { layer size }\end{array}$ & $\begin{array}{c}\text { Number of } \\
\text { hidden layers }\end{array}$ & $\begin{array}{c}\text { Optimizer, } \\
\text { learning rate }\end{array}$ & $\begin{array}{c}\text { Number of } \\
\text { epochs }\end{array}$ & $\begin{array}{c}\text { Batch } \\
\text { size }\end{array}$ & $\begin{array}{c}\text { Activation } \\
\text { function }\end{array}$ & $\begin{array}{c}\text { Weight } \\
\text { initialization }\end{array}$ & Input data-scaling method \\
\hline $2^{6}$ & 10 & $\begin{array}{c}\text { Adam, } \\
1 \mathrm{e}-3\end{array}$ & 1000 & $2^{7}$ & ReLu & He & $\begin{array}{c}\text { No Scaling, } \\
x \text {-Only Scaling, } \\
0 \text { to 1 Min-Max Scaling, } \\
-1 \text { to 1 Min-Max Scaling, } \\
\text { Standard Scaling }\end{array}$ \\
\hline
\end{tabular}

Where the training model with No Scaling means that the input data of $\left\{x, y, u, v, r, \psi, d_{1}, d_{2}\right\}$, where $x, y$ are the actual $x, y$ coordinates of the ship from the berthing facility, are used for the neural network. The training model with the $x y$-Only Scaling means that the input data of $\left\{\eta, \xi, u, v, r, \psi, d_{1}, d_{2}\right\}$ are used for the model. 
In Fig. 6, the significance of application of the input data-scaling method is clearly seen by its training speed and convergence. Standard Scaling shows the fastest training speed and best convergence with the least oscillation, while No Scaling resulted in poor training performance.
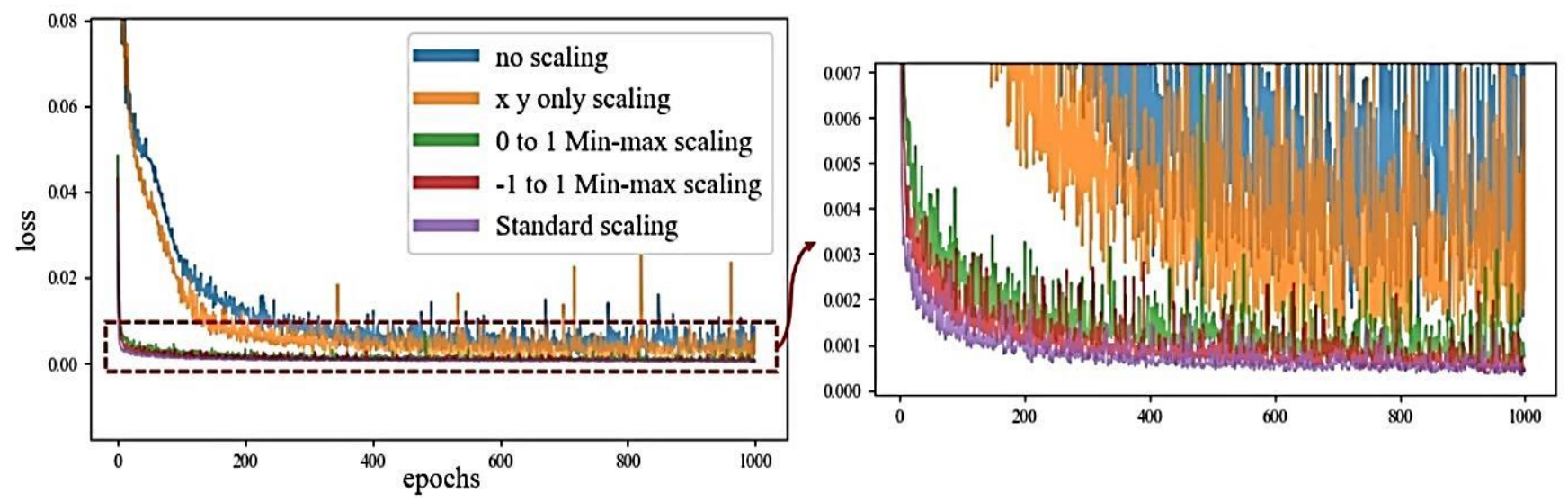

Fig. 6 Loss function histories with respect to input data-scaling methods

Next, the effect of the number of hidden layers is analyzed based on the loss function history and berthing performance history with initial positions from the training dataset. The berthing performance history is obtained as in Algorithm 2.

Algorithm 2 Procedure for obtaining berthing performance history

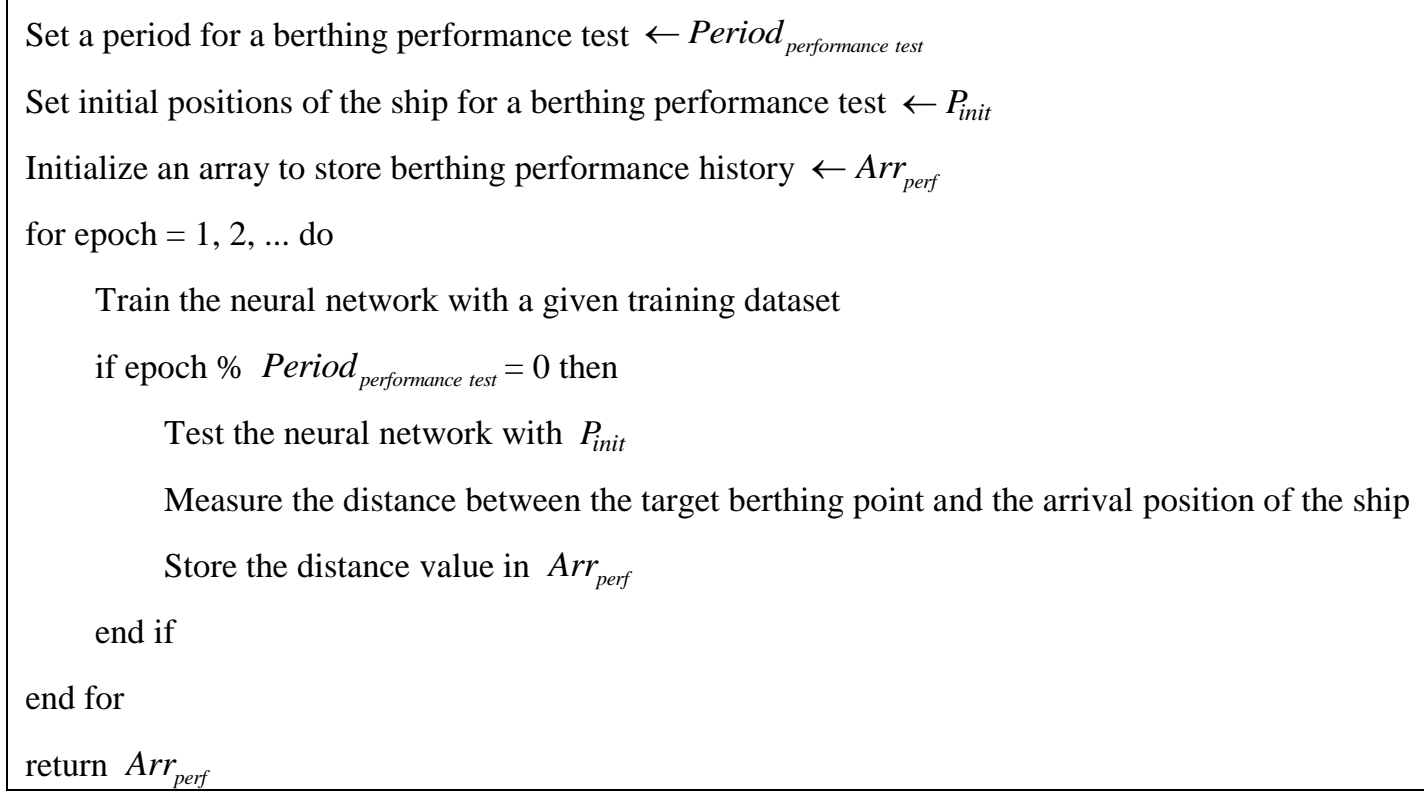

The loss function history and berthing performance history with initial positions of the ship from the training dataset with respect to the number of hidden layers are shown in Fig. 7. Details of the training models are listed in Table 5. In Fig. 7(a), $n \_$hidden_layer of 5 and 10 result in the most stable training, whereas the others show somewhat unstable training with fluctuations. In Fig. 7(b), the $y$-axis of the distance represents the distance gap between the target berthing point and arrival position of the ship, as shown in Algorithm 2. Thus, when the distance is zero, this indicates the highest value and thus the highest performance. The edges of the shaded areas in Fig. 7(b) represent the actual data. It is shown that the training model with the $n$ hidden_layer of 10 shows the highest performance, and an unnecessarily complex neural network model with many hidden layers decreases the performance.

Table 5 Details of training models with respect to number of hidden layers

\begin{tabular}{|c|c|c|c|c|c|c|c|}
\hline $\begin{array}{c}\text { Hidden } \\
\text { layer size }\end{array}$ & $\begin{array}{c}\text { Number of } \\
\text { hidden layers }\end{array}$ & $\begin{array}{c}\text { Optimizer, learning } \\
\text { rate }\end{array}$ & $\begin{array}{c}\text { Number of } \\
\text { epochs }\end{array}$ & Batch size & $\begin{array}{c}\text { Activation } \\
\text { function }\end{array}$ & $\begin{array}{c}\text { Weight } \\
\text { initialization }\end{array}$ & $\begin{array}{c}\text { Input data-scaling } \\
\text { method }\end{array}$ \\
\hline $2^{6}$ & $5,10,20,30,40$ & Adam, 1.0e-3 & 1000 & $2^{7}$ & ReLu & He & Standard Scaling \\
\hline
\end{tabular}




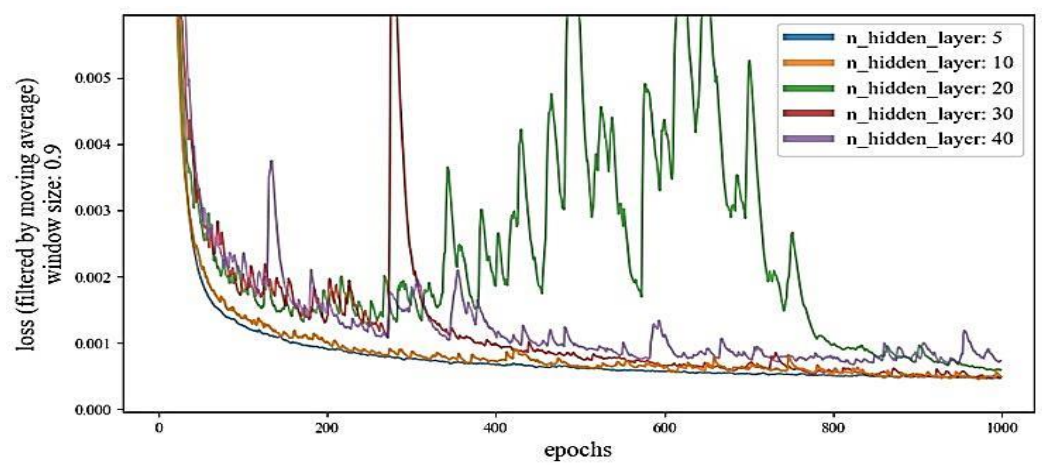

(a) Loss function histories

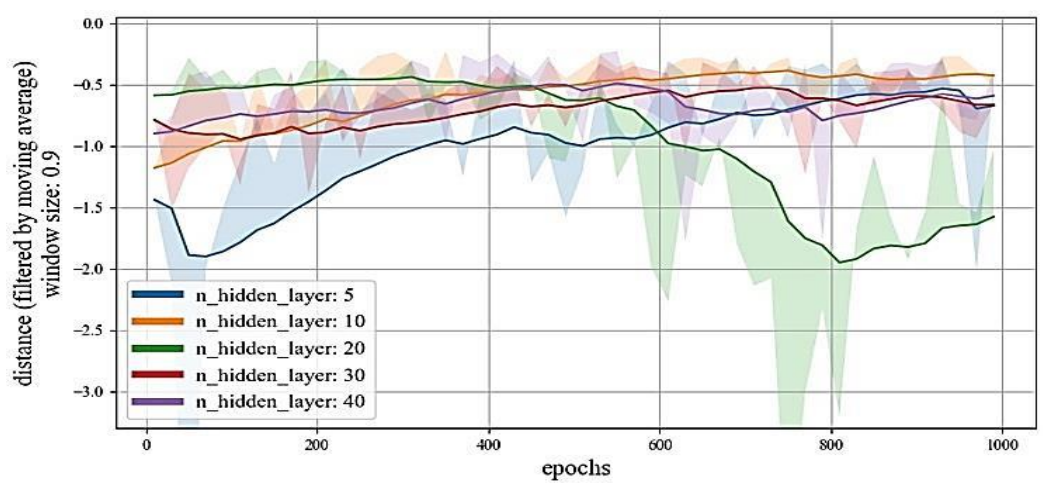

(b) Berthing performance histories with initial positions from training dataset

Fig. 7 Loss function and berthing performance histories with respect to number of hidden layers

Finally, to verify the model's universal berthing performance, the effect of using the BN to prevent overfitting and to improve the neural network's performance is analyzed by the loss function history and berthing performance history with initial positions from the training dataset and a set of new initial positions that are not included in the training dataset. The set of new initial positions is shown in Fig. 8, where the red crosses are the initial positions in the teaching data and the blue ships represent the new initial positions.

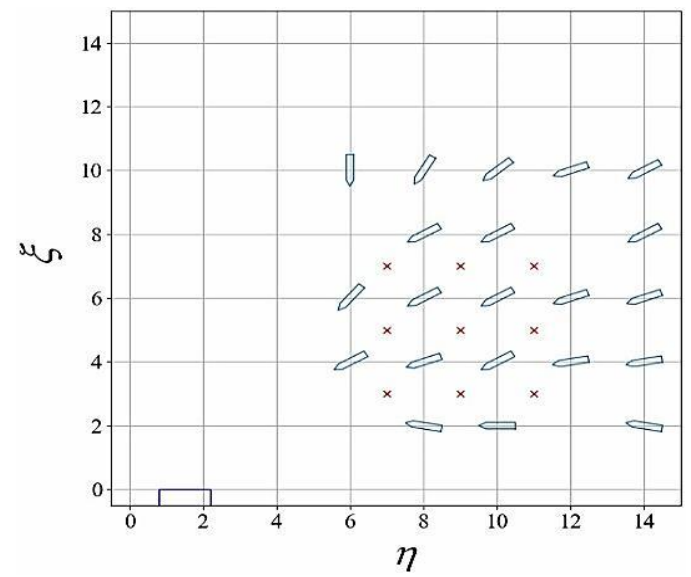

Fig. 8 Set of new initial positions for berthing performance history to verify universal berthing performance

Table 6 Details of training models when BN is used

\begin{tabular}{|c|c|c|c|c|c|c|c|c|}
\hline $\begin{array}{c}\text { hidden } \\
\text { layer } \\
\text { size }\end{array}$ & $\begin{array}{c}\text { number of } \\
\text { hidden layers }\end{array}$ & $\begin{array}{c}\text { optimizer, } \\
\text { learning rate }\end{array}$ & $\begin{array}{c}\text { number of } \\
\text { epochs }\end{array}$ & $\begin{array}{c}\text { batch } \\
\text { size }\end{array}$ & $\begin{array}{c}\text { activation } \\
\text { function }\end{array}$ & $\begin{array}{c}\text { weight } \\
\text { initialization }\end{array}$ & $\begin{array}{c}\text { input } \\
\text { data-scaling } \\
\text { method }\end{array}$ & $\begin{array}{c}\text { use of } \\
\text { BN }\end{array}$ \\
\hline $2^{6}$ & 10 & $\begin{array}{c}\text { Adam, } \\
1 \mathrm{e}-3\end{array}$ & 1000 & $2^{7}$ & ReLu & He & Standard Scaling & $\begin{array}{c}\text { True, } \\
\text { False }\end{array}$ \\
\hline
\end{tabular}

The loss function and berthing performance histories, and details of the training models with respect to the use of the BN, are shown in Fig. 9 and Table 6, respectively. In Fig. 9(a), it can be seen that the BN prevents overfitting and stabilizes the 
training process. The performances in Fig. 9(b) show that the model with BN outperforms the model without BN not only for the initial positions from the training dataset but also for the new initial positions that are not included in the training dataset.

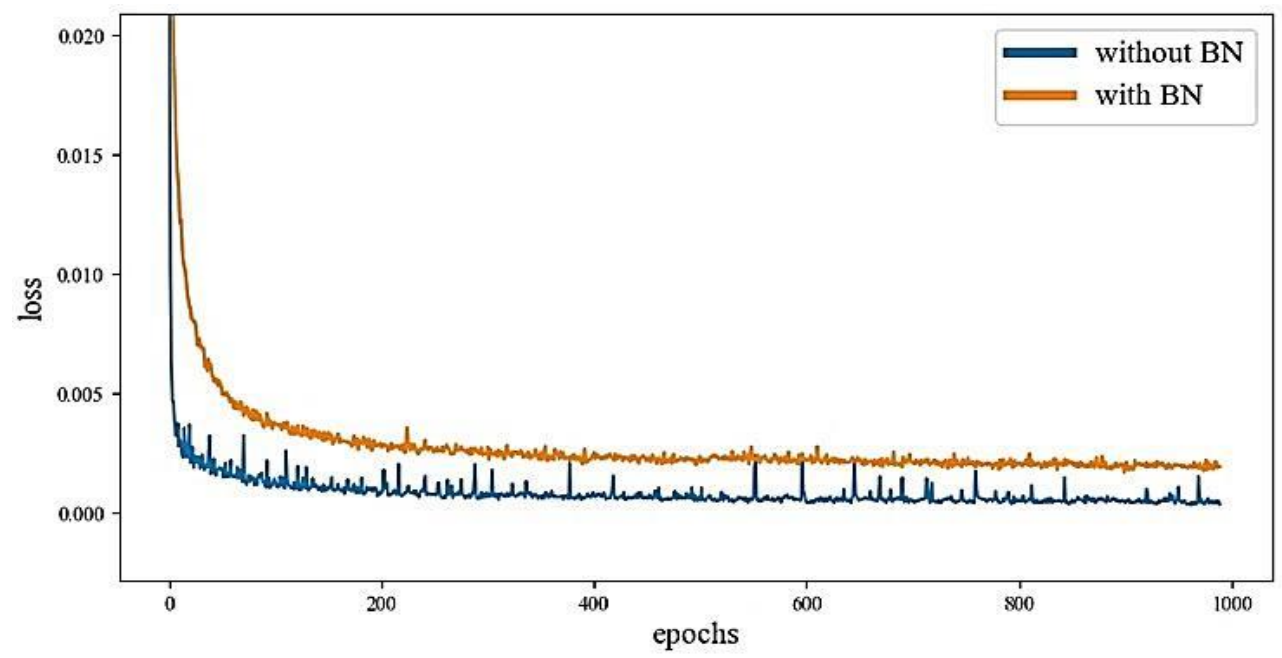

(a) Loss function histories

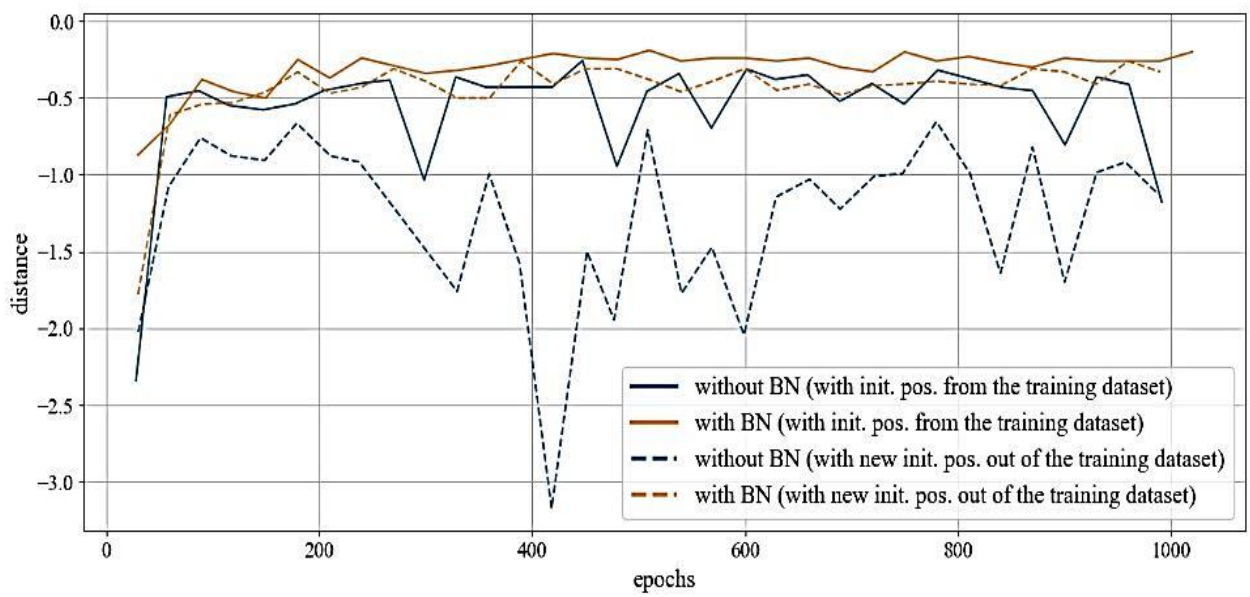

(b) Berthing performance histories

Fig. 9 Loss function and berthing performance histories when $\mathrm{BN}$ is used

Algorithm 3 Trained model selection algorithm for neural networks for automatic ship berthing Initialize an array to store harmonic mean values $\leftarrow A r r_{h m}$

Set the berthing performance history data with the initial positions from the training dataset $\leftarrow X$ Set the berthing performance history data with the new initial positions out of the training dataset $\leftarrow Y$ for each $x \in X$ and $y \in Y$ then

Concatenate $X$ and $Y \leftarrow X Y$

$$
\begin{aligned}
& x^{\prime}=(x-\min (X Y)) /(\max (X Y)-\min (X Y)) / / 0 \text { to } 1 \text { Min-max Scaling } \\
& y^{\prime}=(y-\min (X Y)) /(\max (X Y)-\min (X Y)) \\
& h m=\left(2 x^{\prime} y^{\prime}\right) /\left(x^{\prime}+y^{\prime}\right) \quad / / \text { harmonic mean }
\end{aligned}
$$

Store $\mathrm{hm}$ in $\mathrm{Arr}_{\mathrm{hm}}$ end for Index of the best model is $\arg \max \left(A r r_{h m}\right)$ return $\arg \max \left(A r r_{h m}\right)$ 
Although the trained model with the BN shows good and stable performance over epochs, it is ideal to select the best-trained model of them all. In order to select the best-trained model, a trained model selection algorithm for neural network models for automatic berthing using the harmonic mean is proposed. The trained model selection algorithm is shown in Algorithm 3.

We adopted the harmonic mean to penalize cases where the gap between the berthing performances with the initial positions from and out of the training dataset is high, meaning that the model is overfit to the given training dataset and has a poor universal berthing performance. It has been shown that the use of the recent activation functions, weight initialization method, and input data-scaling methods resulted in faster training speeds and better convergence of the loss function. In addition, the BN improved the actual berthing performance for universal use and stabilized the training process. With the trained model selection algorithm, the two best-trained models were selected for the final simulation in Section 5. One is from the trained models with the $\mathrm{BN}$, and the other is from the trained models without the $\mathrm{BN}$. The significance of the $\mathrm{BN}$ application is shown and discussed in the next section.

\section{Simulation and Results Discussion}

In this section, the berthing trajectories by two different trained models with and without the BN are presented to show how the BN improves the neural network for ship berthing and solves the extrapolation problem. First, berthing trajectories with the initial positions from the training dataset are presented. And then, the berthing trajectories with interpolated and extrapolated initial positions are presented next, respectively. In the berthing simulations in this section, the initial $u$ is set to 1.5 , and the initial $n$ is set to 0.5 for all cases.

To validate if the two trained neural network models perform efficiently when initial positions from a training dataset are given, berthing trajectories with initial positions from a training dataset are presented in Fig. 10. As expected from the berthing performance history in Fig. 9(b), similar berthing performance and trajectories are shown with the initial positions from the training dataset as shown in Fig. 10(a)-(i), meaning that both trained models are trained well for the given training dataset.

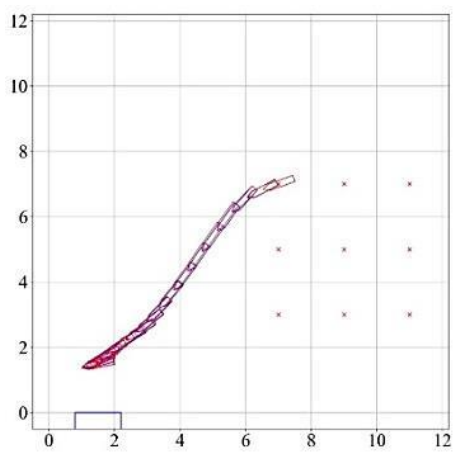

(a) $(\eta, \xi, \psi):\left(7,7,250^{\circ}\right)$

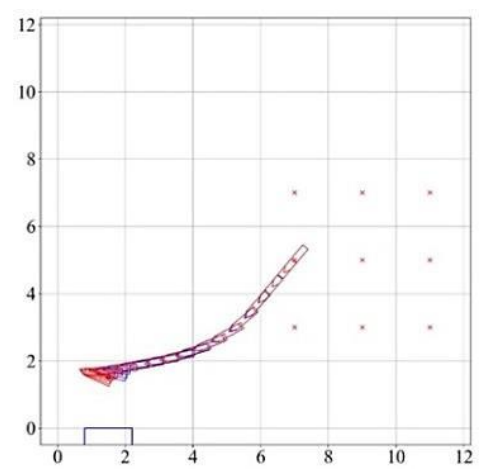

(d) $(\eta, \xi, \psi):\left(7,5,220^{\circ}\right)$

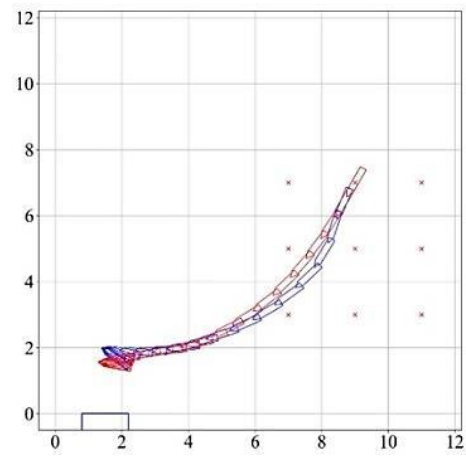

(b) $(\eta, \xi, \psi):\left(9,7,210^{\circ}\right)$

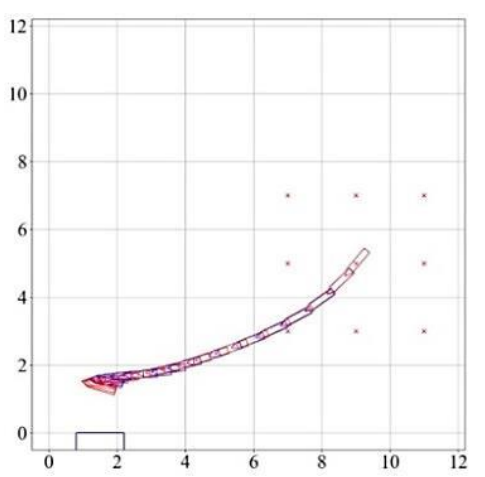

(e) $(\eta, \xi, \psi):\left(9,5,220^{\circ}\right)$

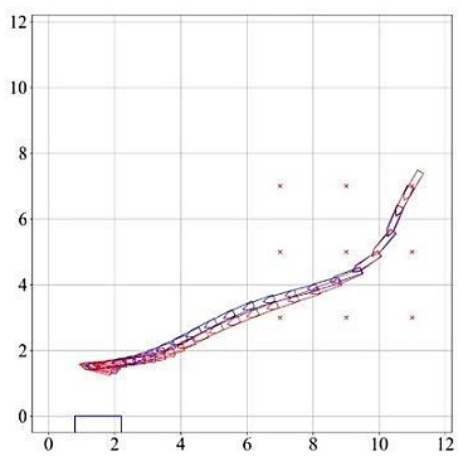

(c) $(\eta, \xi, \psi):\left(11,7,250^{\circ}\right)$

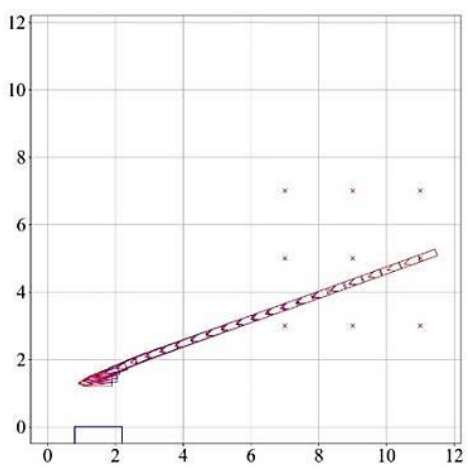

(f) $(\eta, \xi, \psi):\left(11,5,250^{\circ}\right)$

Fig. 10 Berthing trajectories with initial positions from training dataset (red: with BN, blue: without BN) 


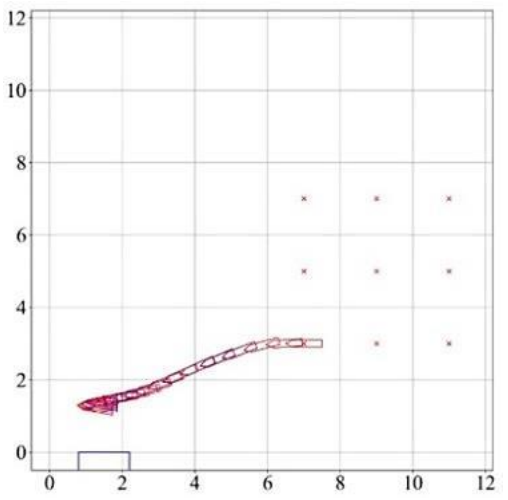

(g) $(\eta, \xi, \psi):\left(7,3,270^{\circ}\right)$

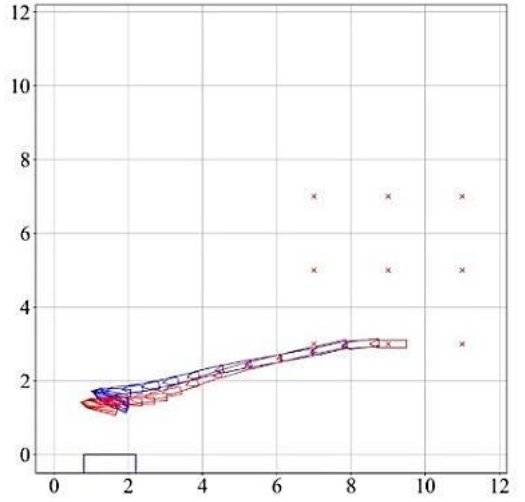

(h) $(\eta, \xi, \psi):\left(9,3,270^{\circ}\right)$

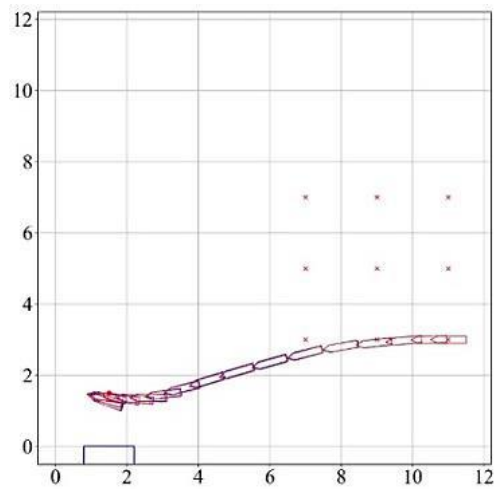

(i) $(\eta, \xi, \psi):\left(11,3,270^{\circ}\right)$

Fig. 10 Berthing trajectories with initial positions from training dataset (red: with BN, blue: without $\mathrm{BN}$ ) (continued)

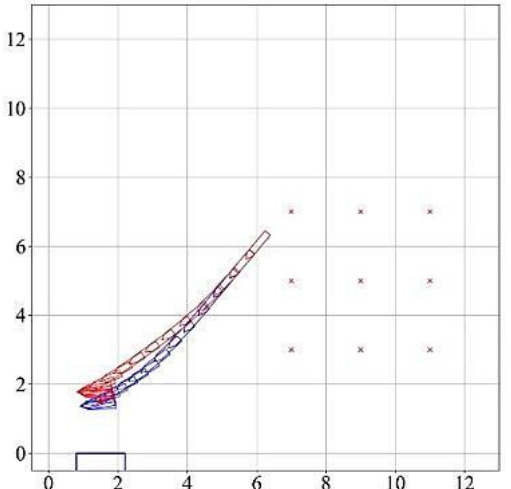

(a) $(\eta, \xi, \psi):\left(6,6,220^{\circ}\right)$

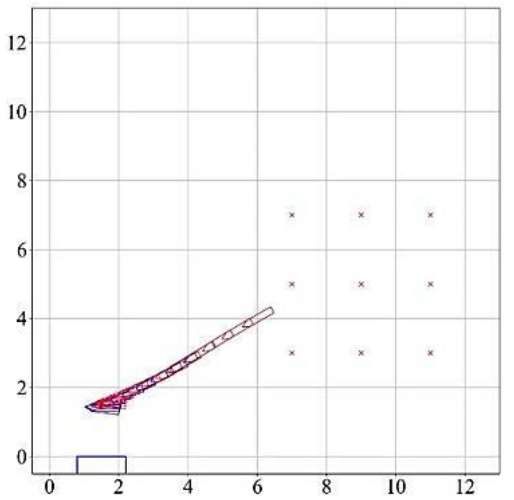

(d) $(\eta, \xi, \psi):\left(6,4,240^{\circ}\right)$

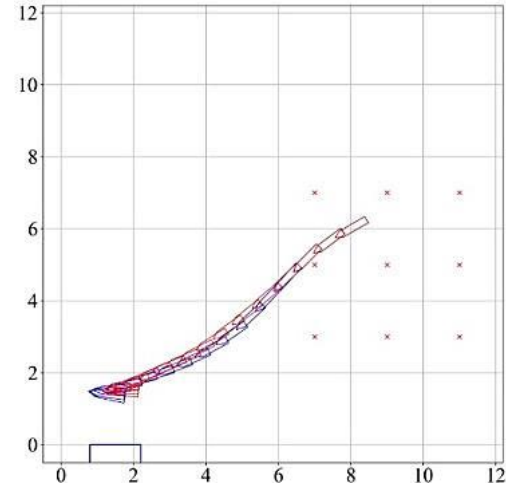

(b) $(\eta, \xi, \psi):\left(8,6,240^{\circ}\right)$

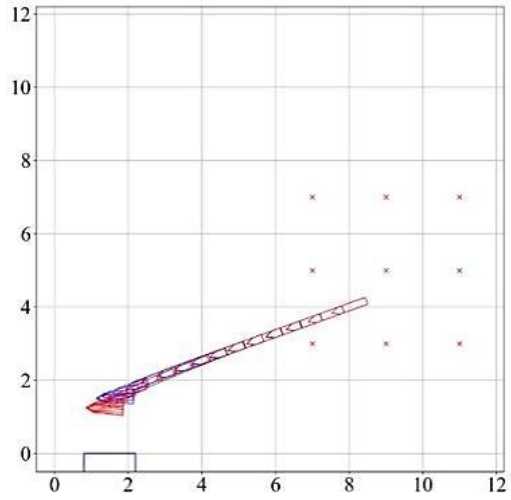

(e) $(\eta, \xi, \psi):\left(8,4,250^{\circ}\right)$

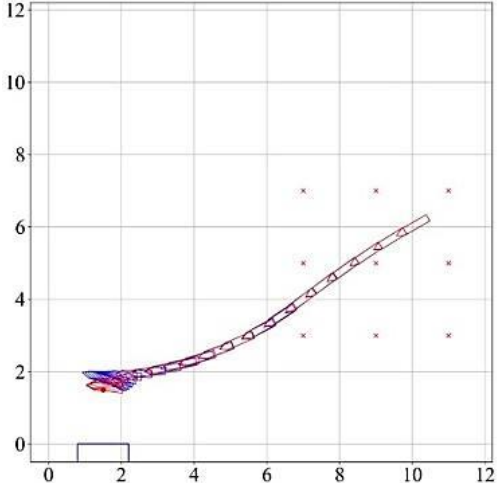

(c) $(\eta, \xi, \psi):\left(10,6,240^{\circ}\right)$

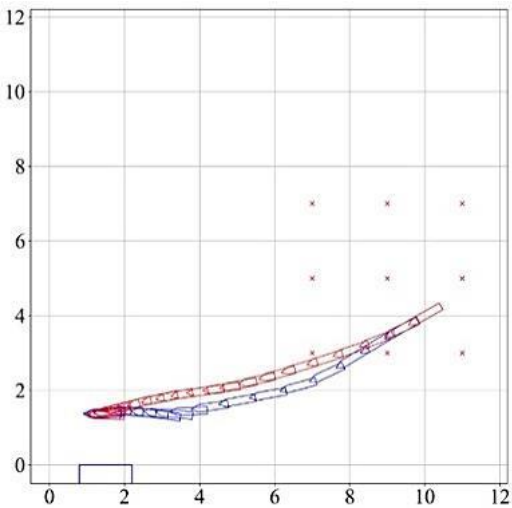

(f) $(\eta, \xi, \psi):\left(10,4,240^{\circ}\right)$

Fig. 11 Berthing trajectories with interpolated initial positions (red: with BN, blue: without BN)

To validate if the two trained neural network models perform efficiently when interpolated initial positions are given, berthing trajectories with the interpolated initial positions are presented in Fig. 11. It can be observed that when the interpolated initial positions are given, both trained models showed successful berthing performances with similar berthing trajectories as shown in Fig. 11(a)-(f)

In the following simulations, the trained models are given three types of extrapolated initial positions. The types of extrapolated initial positions differ by the magnitude of extrapolation.

Berthing trajectories with the first type of extrapolated initial position are shown in Fig. 12. It can be observed that although the trained model without the BN shows good berthing performance in a few cases such as Fig. 12(a)-(b), it also shows poor performance as shown in Fig. 12(c)-(d), while the model with the BN performs successfully in all the cases. In addition, it can be seen that the two trained models control quite differently to reach the target berthing point, as seen in Fig. 12(e)-(f). 


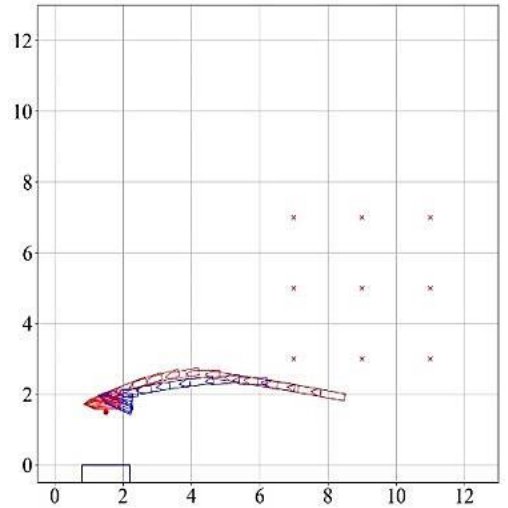

(a) $(\eta, \xi, \psi):\left(8,2,280^{\circ}\right)$

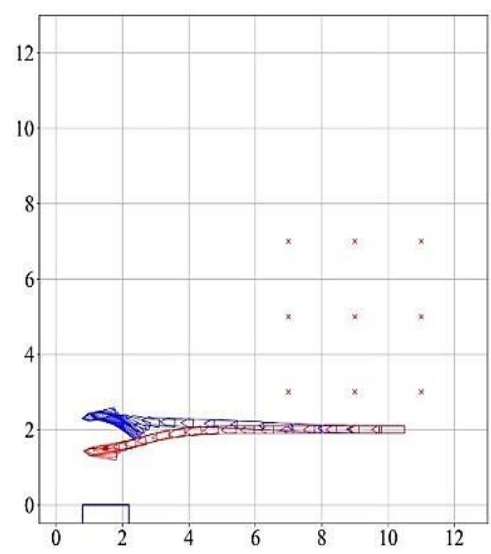

(d) $(\eta, \xi, \psi):\left(10,2,270^{\circ}\right)$

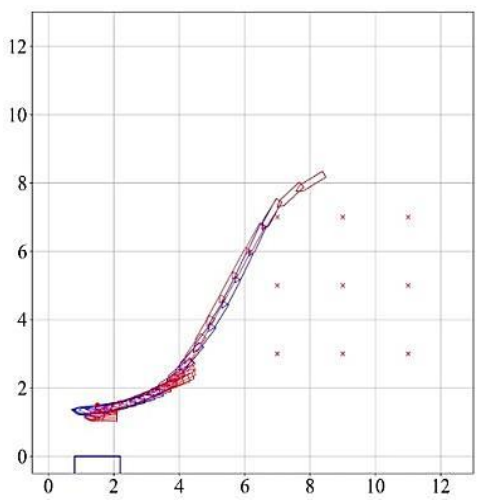

(b) $(\eta, \xi, \psi):\left(8,8,240^{\circ}\right)$

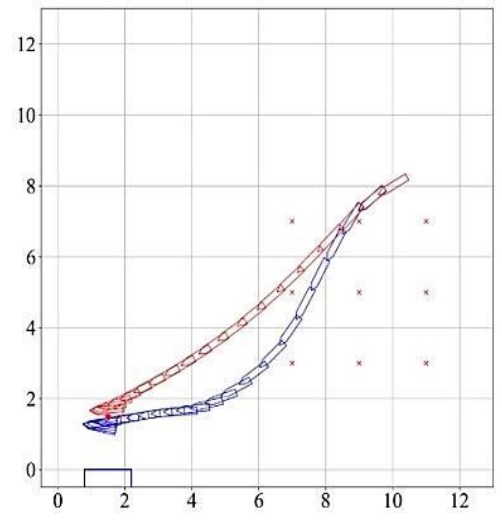

(e) $(\eta, \xi, \psi):\left(10,8,240^{\circ}\right)$

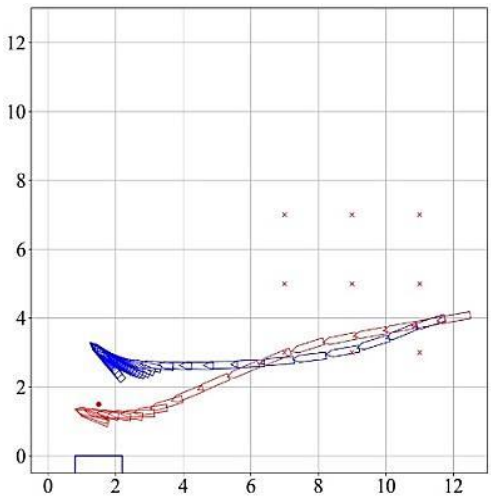

(c) $(\eta, \xi, \psi):\left(12,4,260^{\circ}\right)$

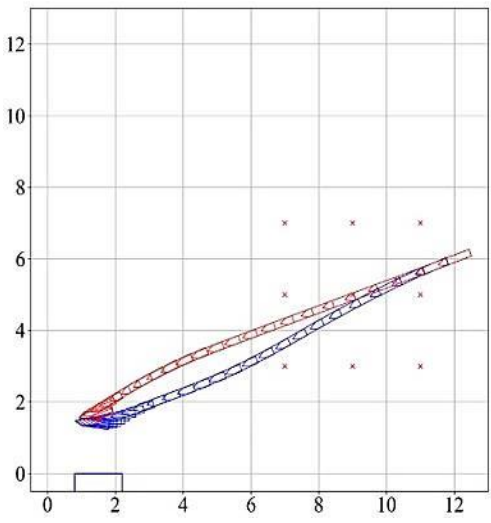

(f) $(\eta, \xi, \psi):\left(12,6,250^{\circ}\right)$

Fig. 12 Berthing trajectories with extrapolated initial positions, Type 1 (red: with BN, blue: without BN)

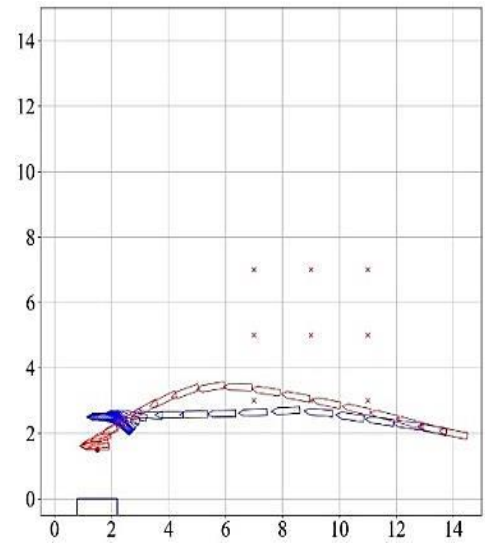

(a) $(\eta, \xi, \psi):\left(14,2,280^{\circ}\right)$

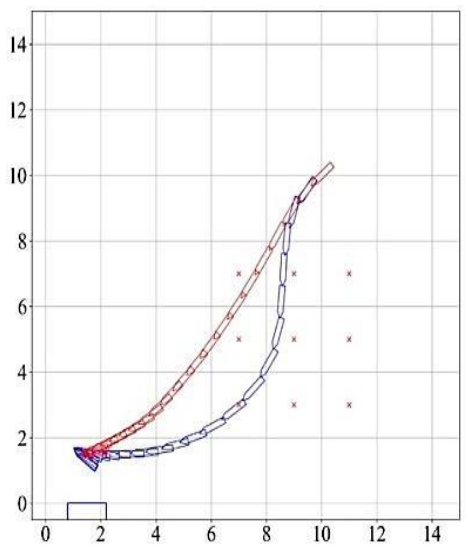

(d) $(\eta, \xi, \psi):\left(10,10,230^{\circ}\right)$

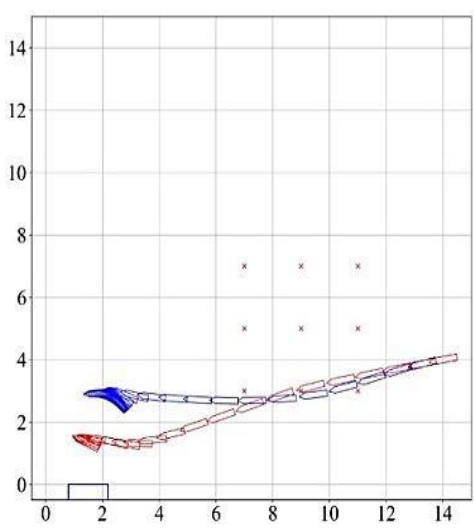

(b) $(\eta, \xi, \psi):\left(14,4,260^{\circ}\right)$

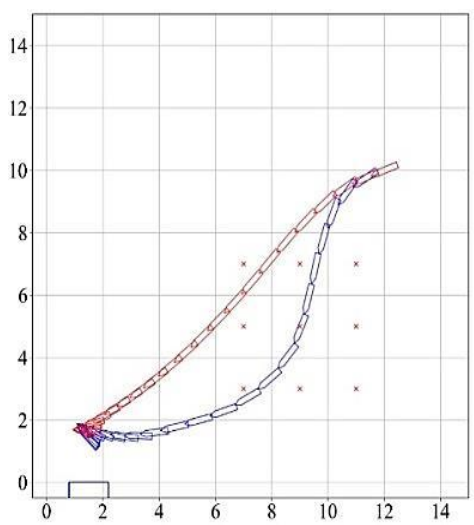

(e) $(\eta, \xi, \psi):\left(12,10,250^{\circ}\right)$

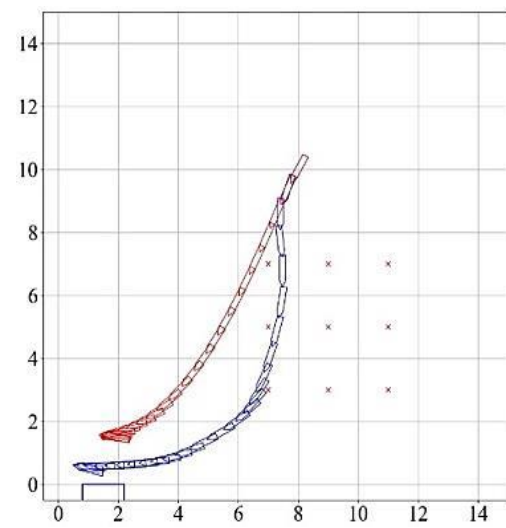

(c) $(\eta, \xi, \psi):\left(8,10,210^{\circ}\right)$

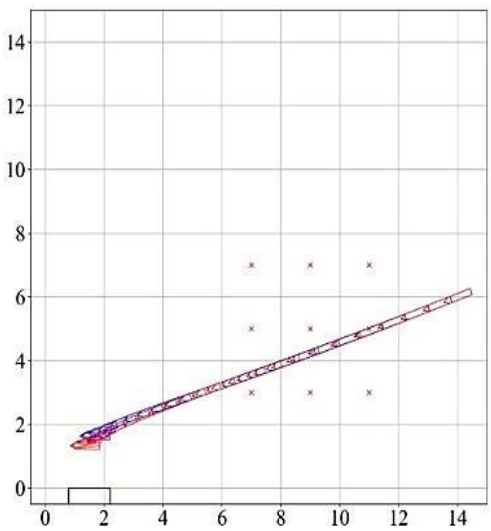

(f) $(\eta, \xi, \psi):\left(14,6,250^{\circ}\right)$

Fig. 13 Berthing trajectories with extrapolated initial positions, Type 2 (red: with BN, blue: without BN) 
Next, berthing trajectories with the second type of extrapolated initial position are shown in Fig. 13. As the more extrapolated initial positions are given in the cases in Fig. 13, the trained model without the BN suffers from the extrapolation issue even more as shown in Fig. 13(a)-(e). The only case where the trained model without the BN performs well is when it berths in a straight line as shown in Fig. 13(f).

Finally, berthing trajectories with the third type of extrapolated initial position are shown in Fig. 14, in which the extrapolation of initial positions is the greatest in order to clearly see the effect of the BN. In Fig. 14. The model without the BN performs poorly overall owing to the extrapolation problem. The effectiveness of application of the $\mathrm{BN}$ is clear, as the BN improves the neural network model for more general use.

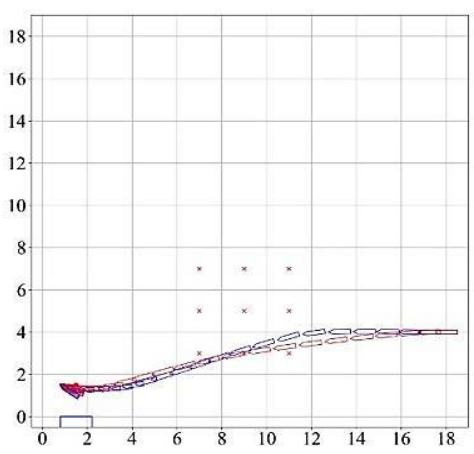

(a) $(\eta, \xi, \psi):\left(18,4,270^{\circ}\right)$

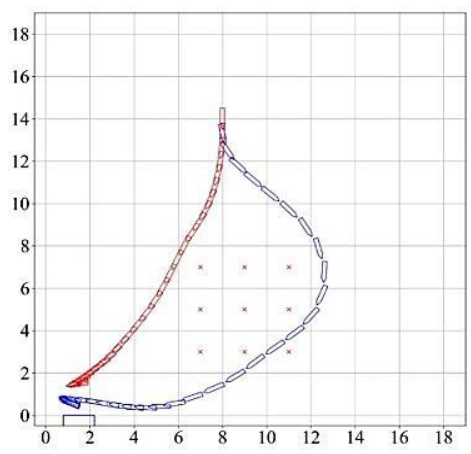

(d) $(\eta, \xi, \psi):\left(8,14,180^{\circ}\right)$

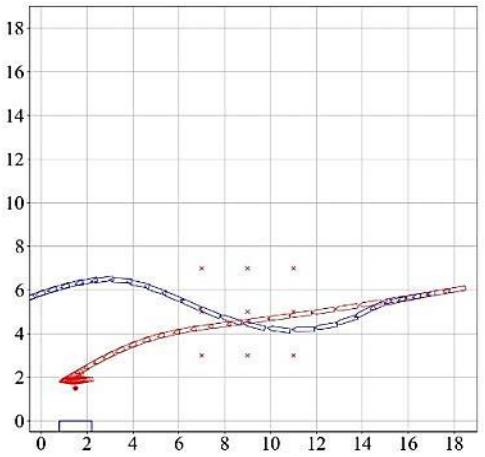

(b) $(\eta, \xi, \psi):\left(14,6,260^{\circ}\right)$

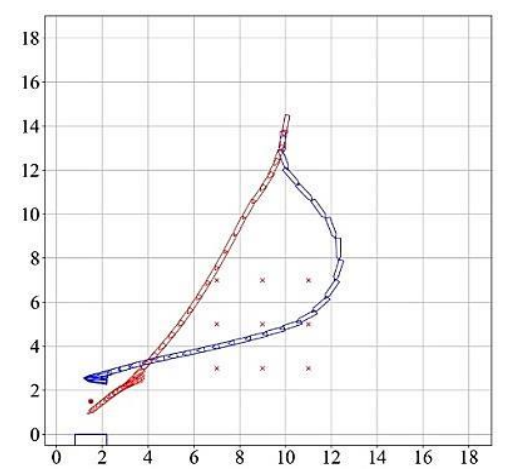

(e) $(\eta, \xi, \psi):\left(10,14,190^{\circ}\right)$

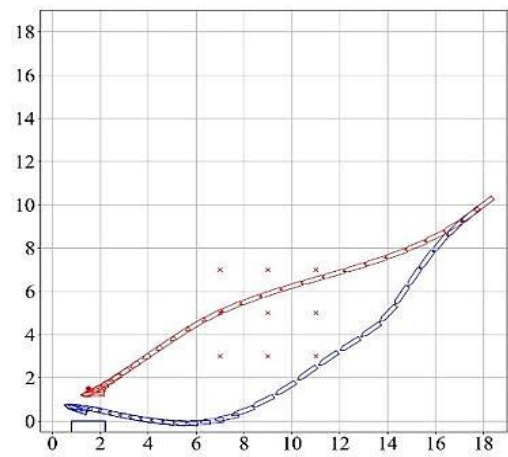

(c) $(\eta, \xi, \psi):\left(18,6,230^{\circ}\right)$

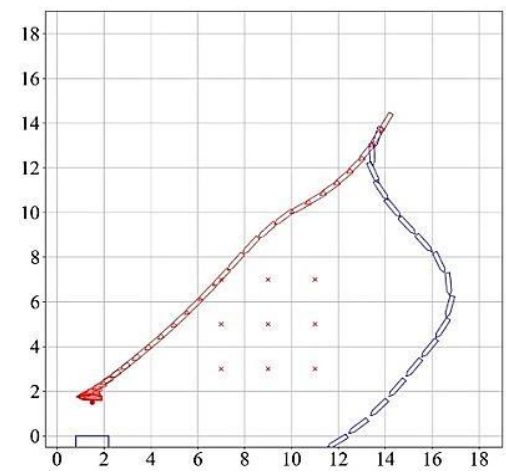

(f) $(\eta, \xi, \psi):\left(14,14,210^{\circ}\right)$

Fig. 14 Berthing trajectories with extrapolated initial positions, Type 3 (red: with BN, blue: without BN)

\section{Conclusion}

We showed that with the application of recent activation functions, weight initialization methods, input data-scaling methods, and a higher number of hidden layers, faster training speed and better training convergence can be achieved. In order to observe the progress of the berthing performance over epochs and select the best-trained model, the algorithm for obtaining the berthing performance history and the model selection algorithm were proposed as Algorithms 2 and 3, respectively. Last, the use of the BN can stabilize the training process and solve the extrapolation problem by preventing overfitting. A neural network model with the BN was able to perform successfully, not only with interpolated and slightly extrapolated initial positions but also with greatly extrapolated initial positions. This makes the neural network models more universal for automatic berthing.

\section{Acknowledgement}

This material is based upon work supported by the Ministry of Trade, Industry \& Energy (MOTIE, Korea) under Industrial Technology Innovation Program. No. 10063405, "Development of hull form of year-round floating-type offshore structure based on the Arctic Ocean in ARC7 condition with dynamic positioning and mooring system." 


\section{Conflicts of Interest}

The authors declare no conflict of interest.

\section{References}

[1] N. K. Im and K. Hasegawa, "Automatic ship berthing using parallel neural controller," Proc. International Federation of Automatic Control (IFAC), vol. 34, no. 7, pp. 51-57, 2001.

[2] N. K. Im and K. Hasegawa, "Motion identification using neural networks and its application to automatic ship berthing under wind," Journal of Ship and Ocean Technology, vol. 6, no. 1, pp. 16-26, 2002.

[3] N. K. Im, "All direction approach automatic ship berthing controller using ANN (artificial neural networks)," Journal of Institute of Control, Robotics and Systems, vol. 13, no. 4, pp. 304-308, 2007.

[4] C. H. Bae, S. K. Lee, S. E. Lee, and J. H. Kim, "A study of the automatic berthing system of a ship using artificial neural network," Journal of Korean Navigation and Port Research, vol. 32, no. 8, pp. 589-596, 2008.

[5] Y. A. Ahmed and K. Hasegawa, "Automatic ship berthing using artificial neural network trained by consistent teaching data using nonlinear programming method," Engineering Applications of Artificial Intelligence, vol. 26, no. 10, pp. 2287-2304, 2013.

[6] N. K. Im and V. S. Nguyen, "Artificial neural network controller for automatic ship berthing using head-up coordinate system," International Journal of Naval Architecture and Ocean Engineering, vol. 10, no. 3, pp. 235-249, 2018.

[7] V. Nair and G. E. Hinton, "Rectified linear units improve restricted Boltzmann machines," Proc. 27th International Conference on Machine Learning (ICML-10), 2010.

[8] A. L. Maas, A. Y. Hannun, and A. Y. Ng, "Rectifier nonlinearities improve neural network acoustic models," Proc. International Conference on Machine Learning, vol. 30, no. 1, 2013.

[9] K. Levenberg, "A method for the solution of certain non-linear problems in least squares," Quarterly of Applied Mathematics, vol. 2, no. 2, pp. 164-168, 1944.

[10] T. Bazzi, R. Ismail, and M. Zohdy, "Comparative performance of several recent supervised learning algorithms," International Journal of Computer and Information Technology, vol. 7, 2018.

[11] T. Tieleman and G. Hinton, Lecture 6.5-rmsprop: Divide the gradient by a running average of its recent magnitude, COURSERA: Neural Networks for Machine Learning, 2012.

[12] D. P. Kingma and J. Ba, “Adam: a method for stochastic optimization,” arXiv preprint arXiv:1412.6980, 2014.

[13] K. H. Shon, "Hydrodynamic forces and maneuvering characteristics of ships at low advance speed," Journal of the Society of Naval Architects of Korea, vol. 29, no. 3, pp. 90-101, 1992.

[14] V. S. Nguyen, V. C. Do, and N. K. Im, "Development of automatic ship berthing system using artificial neural network and distance measurement system," International Journal of Fuzzy Logic and Intelligent Systems, vol. 18, no. 1, pp. 41-49, 2018.

[15] Y. A. Ahmed and K. Hasegawa, "Automatic ship berthing using artificial neural network based on virtual window concept in wind condition," Proc. International Federation of Automatic Control (IFAC), vol. 45, no. 24, pp. 286-291, 2012.

[16] X. Glorot and Y. Bengio, "Understanding the difficulty of training deep feedforward neural network," Proc. International Conference on Artificial Intelligence and Statistics, vol. 9, pp. 246-266, 2010.

[17] K. He, X. Zhang, S. Ren, and J. Sun, "Delving deep into rectifiers: surpassing human-level performance on Imagenet classification," Proc. IEEE International Conference on Computer Vision, pp. 1026-1034, 2015.

[18] H. Robbins and S. Monro, "A stochastic approximation method," The Annals of Mathematical Stastics, pp. 400-407, 1951.

[19] D. E. Rumelhart, G. E. Hinton, and R. J. Williams, "Learning representations by back-propagating errors," Nature, vol. 323, no. 6088 , pp. 533-536, 1986.

[20] J. Duchi, E. Hazan, and Y. Singer, “Adaptive subgradient methods for online learning and stochastic optimization," Journal of Machine Learning Research, pp. 2121-2159, July 2011.

[21] C. M. Bishop, Neural networks for pattern recognition. Oxford: Clarendon Press, New York, 1996.

[22] N. Srivastava, G. Hinton, A. Krizhevsky, I. Sutskever, and D. Salakhutdinov, "Dropout: A simple way to prevent neural networks from overfitting," Journal of Machine Learning Research, vol. 15, no.1, pp. 1929-1958, 2014.

[23] S. Loffe and C. Szegedy, "Batch normalization: Accelerating deep network training by reducing internal covariate shift," arXiv preprint arXiv:1502.03167, 2015.

[24] J. N. Newman, Marine hydrodynamics. Cambridge, MA: MIT Press, 1980.

[25] C. Nwankpa, W. Ijomah, A. Gachagan, and S. Marshall, "Activation functions: comparison of trends in practice and research for deep learning," arXiv preprint arXiv:1811.03378, 2018.

[26] S. K. Lee, G. W. Lee, S. J. Lee, and S. R. Jeong, "A study on the automatic berthing control of a ship by artificial neural network," Journal of Korean Navigation and Port Research, vol. 32, no. 4, pp. 21-28, 1997. 